\title{
Robot-assisted gait training to reduce pusher behavior
}

\section{A randomized controlled trial}

Jeannine Bergmann, PhD, Carmen Krewer, PhD, Klaus Jahn, MD, and Friedemann Müller, MD

Neurology ${ }^{\circledR}$ 2018;91:e1319-e1327. doi:10.1212/WNL.0000000000006276

\section{Abstract}

\section{Objective}

To determine the effects of 2 weeks of intensive robot-assisted gait training (RAGT) on pusher behavior compared to nonrobotic physiotherapy (nR-PT).

\section{Methods}

In a single-blind, randomized, controlled trial with 2 parallel arms, we compared 2 weeks of daily RAGT (intervention group) with the same amount of nR-PT (control group). Patients with subacute stroke who had pusher behavior according to the Scale for Contraversive Pushing (SCP) were included. The primary research questions were whether changes in pusher behavior would differ between groups post intervention, and at a follow-up 2 weeks afterward (SCP and Burke Lateropulsion Scale, Class II evidence). Secondary outcomes included the Performance-Oriented Mobility Assessment, the Functional Ambulation Classification, and the Subjective Visual Vertical.

\section{Results}

Thirty-eight patients were randomized. Thereof, 30 patients received the allocated intervention and were included in the analyses. RAGT led to a larger reduction of pusher behavior than $\mathrm{nR}$ PT at post test (SCP: $U=69.00, r=-0.33, p=0.037$; Burke Lateropulsion Scale: $U=47.500$, $r=-0.50, p=0.003$ ) and at follow-up (SCP: $U=54.00, r=-0.44, p=0.008$ ). Pusher behavior had ceased in 6 of 15 participants in the intervention group and 1 of 15 participants in the control group at post test. At follow-up, 9 of 15 and 5 of 15 participants, respectively, no longer exhibited the behavior.

\section{Conclusions}

Two weeks of RAGT seems to persistently reduce pusher behavior, possibly by recalibrating the disturbed inner reference of verticality. The potential benefits of RAGT on pusher behavior and verticality perception require further investigation.

\section{Trial registration}

German Clinical Trials Register (registration number: DRKS00003444).

\section{Classification of evidence}

This study provides Class II evidence that RAGT is beneficial to reduce pusher behavior in patients with stroke.

\author{
Correspondence \\ Dr. Bergmann \\ jbergmann@ \\ Schoen-Kliniken.de
}

\section{MORE ONLINE}

$\rightarrow$ Class of Evidence

Criteria for rating

therapeutic and diagnostic studies

NPub.org/coe 


\section{Glossary}

ACE-R = Addenbrooke's Cognitive Examination-Revised; BLS = Burke Lateropulsion Scale; FAC = Functional Ambulation Classification; nR-PT = nonrobotic physiotherapy; POMA = Performance-Oriented Mobility Assessment; POMA-B = Performance-Oriented Mobility Assessment-balance items; RAGT = robot-assisted gait training; SCP = Scale for Contraversive Pushing; SVV = Subjective Visual Vertical.

Pusher behavior is a severe postural disorder exhibited by some patients with stroke and reflects an altered perception of body orientation in space. ${ }^{1-3}$ Patients with pusher behavior typically push themselves away from their nonparetic body side and resist any attempt to transfer weight over to the nonparetic side. ${ }^{4}$ The behavior affects $10 \%$ to $18 \%$ of patients in stroke rehabilitation and considerably hampers the therapy and prolongs the rehabilitation process. $^{5-8}$ Such patients are only half as efficient and effective in their rehabilitation outcome compared to patients with stroke without pusher behavior. ${ }^{8}$ Consequently, innovative treatment approaches that effectively reduce pusher behavior are urgently needed.

So far, rehabilitation approaches for pusher behavior for the most part focused on different forms of feedback training, which means training of postural control strategies by using visual cues, for example. ${ }^{9,10}$ However, evidence on the effectiveness of feedback training is insufficient, and patients seem often unable to use the cues spontaneously. In a pilot study, the immediate effects of a single session of physiotherapy with visual feedback components, galvanic vestibular stimulation, and robot-assisted gait training (RAGT) were compared. ${ }^{11}$ After a session of RAGT, patients showed a statistically significant reduction in pusher behavior compared to physiotherapy, while galvanic vestibular stimulation did not cause any statistically significant improvements. Forced control of upright body position during locomotion seems to be an effective method for immediately reducing pusher behavior.

The objective of this randomized controlled trial was to determine the effects of 2 weeks of intensive RAGT on pusher behavior in comparison to physiotherapy without robotic support.

\section{Methods}

This was a single-blind, randomized, controlled trial with 2 parallel arms.

\section{Standard protocol approvals, registrations, and patient consents}

The study was approved by the ethics committee of the Ludwig-Maximilians University Munich. All participants or their legal representatives gave their written informed consent. This trial was registered at the German Clinical Trials Register (DRKS00003444).

\section{Participants}

All participants included in the study were inpatients in a rehabilitation setting at the Schön Klinik Bad Aibling. They met the following inclusion criteria: hemiparesis after first unilateral ischemic or hemorrhagic stroke, 3 weeks to 6 months since onset, age between 18 and 90 years, pusher behavior (Scale for Contraversive Pushing [SCP] $>0$ per component, assessed by a physiotherapist and the study coordinator), and orthostatic tolerance for 30 minutes of passive standing. Patients with extreme osteoporosis, unstable fracture, or excessive spasticity were excluded from the study. Other exclusion criteria were acute diseases of the cardiovascular or respiratory system, and pressure sores on the lower extremities. Body weight was limited to $130 \mathrm{~kg}$, body height to 200 $\mathrm{cm}$, and the maximum leg length difference was $2 \mathrm{~cm}$.

Because patients with pusher behavior frequently exhibit cognitive deficits that may influence the recovery of pusher behavior, ${ }^{12,13}$ the cognitive function was assessed at the beginning of the study with the Addenbrooke's Cognitive Examination-Revised (ACE-R). ${ }^{14}$

\section{Study protocol}

Patients included in the study were randomly allocated to either the intervention group (RAGT) or the control group (nonrobotic physiotherapy [nR-PT]). The randomization sequence was computer-generated with a ratio of $1: 1$ and done by a scientific staff member not directly involved in the project to ensure concealment. This staff member prepared numbered and sealed opaque envelopes that were used by the study coordinator to assign participants after study inclusion.

During the intervention phase, patients performed 8 to 10 sessions of the respective therapeutic intervention. The intervention was planned to occur on 5 days per week for 2 weeks. If a patient was not able to finish all sessions as planned, up to 2 additional sessions were scheduled in the following week in order to have values from at least 8 sessions for each patient. Primary and secondary outcome measures were assessed before the intervention phase (baseline), at the end of the 2-week intervention phase (post test), and at a followup test 2 weeks after the end of the intervention phase. During the follow-up phase, no RAGT or treadmill training was performed until the follow-up test.

\section{Interventions}

Patients of the intervention group received RAGT on the robotic-driven gait orthosis Lokomat (Hocoma AG, Volketswil, Switzerland). A RAGT session lasted 60 minutes. The 
Lokomat is an exoskeleton with linear drives on hip and knee joints that assist locomotion on a treadmill by guiding the participant's legs along a predefined trajectory. Patients are connected to the gait orthosis by a harness, which is attached to a body-weight support system, and by cuffs placed around the legs. Elastic straps are used to passively lift the participant's feet and prevent foot drop. Body weight support was individually set for each patient, but amounted to no more than $50 \%$ of the patient's body weight. Guidance force was set at $100 \%$ on both sides. After a short warming-up period, walking speed was increased to $2 \mathrm{~km} / \mathrm{h}$ or faster. The target walking time was at least 20 minutes.

Patients of the control group received nR-PT. A session of nRPT was scheduled for 60 minutes, either $2 \times 30$ minutes or $1 \times$ 30 minutes with cotherapy ( 2 therapists; the target was at least 20 minutes of active therapy). The focus of nR-PT was on training of postural control including sensory feedback components. The therapy consisted only of active and dynamic exercises, such as shifting of the center of gravity; no passive or static exercises were planned. Therapists and patients were allowed to use external references, such as a wall or a handrail on the nonparetic side, and visual feedback, such as the door frame or a mirror. The training was performed while sitting or standing; movement transitions, such as transferring from sitting to standing, and walking if possible, were practiced.

Content and characteristics of each therapy session were documented for both interventions in standardized protocols.

\section{Primary outcome measures}

The primary objective of the study was to determine the effects of RAGT on pusher behavior. Pusher behavior was assessed by using the SCP and the Burke Lateropulsion Scale (BLS). In the preceding pilot study, only the BLS showed statistically significant effects of RAGT. ${ }^{11}$ However, by the time the present study was designed, the SCP was the most frequently used and investigated scale for pusher behavior, while the BLS was seldom evaluated. That is why we decided to use both scales as primary outcome measures. The scales were assessed at baseline, at post test, and at a follow-up test 2 weeks after the end of the intervention phase by a trained assessor who was blinded to treatment allocation.

The SCP has 3 components: (1) the symmetry of spontaneous body posture, (2) the use of nonparetic extremities (leg and arm), and (3) the resistance to passive correction of the tilted posture. ${ }^{2}$ Each component is tested in sitting and standing positions, yielding a maximum score of 2 per component ( 1 for sitting and 1 for standing). The total score of the SCP ranges from 0 to 6 . The SCP has a high interobserver reliability and internal consistency, and a moderate to high construct validity. ${ }^{7,15}$ For the diagnosis of pusher behavior, a cutoff score $>0$ was used for each component. ${ }^{7}$

The BLS rates the patient's resistance to passive supine rolling, to passive postural correction when sitting and standing, and to assistance during transferring and walking. The greater the resistance, the higher the score. ${ }^{16}$ The maximal score of the BLS is 17 . The cutoff for the diagnosis of pusher behavior is $\geq 2$ points. ${ }^{17}$ The BLS shows a high interrater and intrarater reliability. ${ }^{16}$

\section{Secondary outcome measures}

Secondary outcome measures were the balance items of the Performance-Oriented Mobility Assessment (POMA-B), the Functional Ambulation Classification (FAC), and the Subjective Visual Vertical (SVV). The measures were assessed at baseline, at post test, and at a follow-up test 2 weeks after the end of the intervention phase by a trained assessor who was blinded to treatment allocation.

The POMA-B evaluates balance. ${ }^{18}$ It tests different positions and changes in positions, reflecting stability tasks that are related to daily activities. The POMA-B shows high interrater and intrarater reliability and satisfactory concurrent and discriminant validity.

The FAC rates walking ability. It distinguishes 6 levels of walking ability on the basis of the amount of physical support required. ${ }^{19}$ Categories range from 0 (patient cannot walk or needs help from 2 or more persons) to 5 (patient can walk independently anywhere). The FAC has high reliability, good concurrent and predictive validity, and good responsiveness. ${ }^{20}$

The SVV measures a person's perception of visual verticality. The so-called bucket test was used for SVV assessment. ${ }^{21}$ The bucket was rotated by the examiner, and the seated participants indicated when they visually perceived a dark line to be vertical. Six trials were performed in the frontal plane. Results of these trials were averaged to obtain the SVV.

\section{Statistical analysis}

The primary hypothesis was that patients assigned to RAGT would have a larger improvement in pusher behavior (SCP and BLS) than patients assigned to nR-PT after the 2-week intervention phase and at a follow-up (Class II evidence).

An a priori sample-size calculation was performed using the method derived by Noether. ${ }^{22}$ Effect size was estimated based on the data of the previous pilot study resulting in $p_{\text {Noether }}=$ 0.8 for the BLS. ${ }^{11,23}$ Assuming this effect size with a 2-sided significance level of $0.05 \%$ and $80 \%$ power, sample-size calculation resulted in a sample size of 15 patients per group. To account for an anticipated dropout rate of $25 \%$, the minimum number of patients required to enroll per group was increased to 38 patients for the entire study.

Demographic and clinical characteristics of patients were compared between the intervention and the control groups using the $\chi^{2}$ test (for comparison of proportions) or Student $t$ test (for continuous variables). Kendall tau statistics was used to investigate the relationship between the ACE-R score and patients' response to the interventions. 
Data of all patients who received at least one session of the allocated intervention were included in the analyses. Missing outcome values were replaced using the last-observationcarried-forward method.

Nonparametric statistics were used for all outcome variables because of the ordinal scale quality of the measures (SCP, BLS, POMA, FAC), or in case of abnormally distributed data (SVV). Between-group comparisons (intervention vs control group) for the primary and secondary outcome measures were performed for the baseline values, and for the intraindividual differences calculated from baseline to post (shortterm effect) and from baseline to follow-up (long-term effect) using Mann-Whitney $U$ tests. In addition, within-group comparisons were performed using Friedman tests. If the Friedman test showed statistically significant differences, Wilcoxon matched-pairs tests were performed to compare baseline and post, and baseline and follow-up measures. Effect sizes $(r)$ of changes between groups and within-groups were calculated. ${ }^{24}$ Effect size of $0.1,0.3$, and 0.5 represents a small, medium, and large effect, respectively. ${ }^{25}$

The statistical analyses were performed using SPSS Statistics (version 19.0; IBM Corp., Armonk, NY). The significance level for $\alpha$ was set at 0.05 .

\section{Classification of evidence}

This study provides Class II evidence that 2 weeks of intensive RAGT reduces pusher behavior (SCP and BLS) in patients with stroke compared to the same amount of nR-PT.

\section{Data availability}

Datasets generated and analyzed during the current study are available in an anonymized form from the corresponding author on reasonable request.

Figure Participant flow diagram

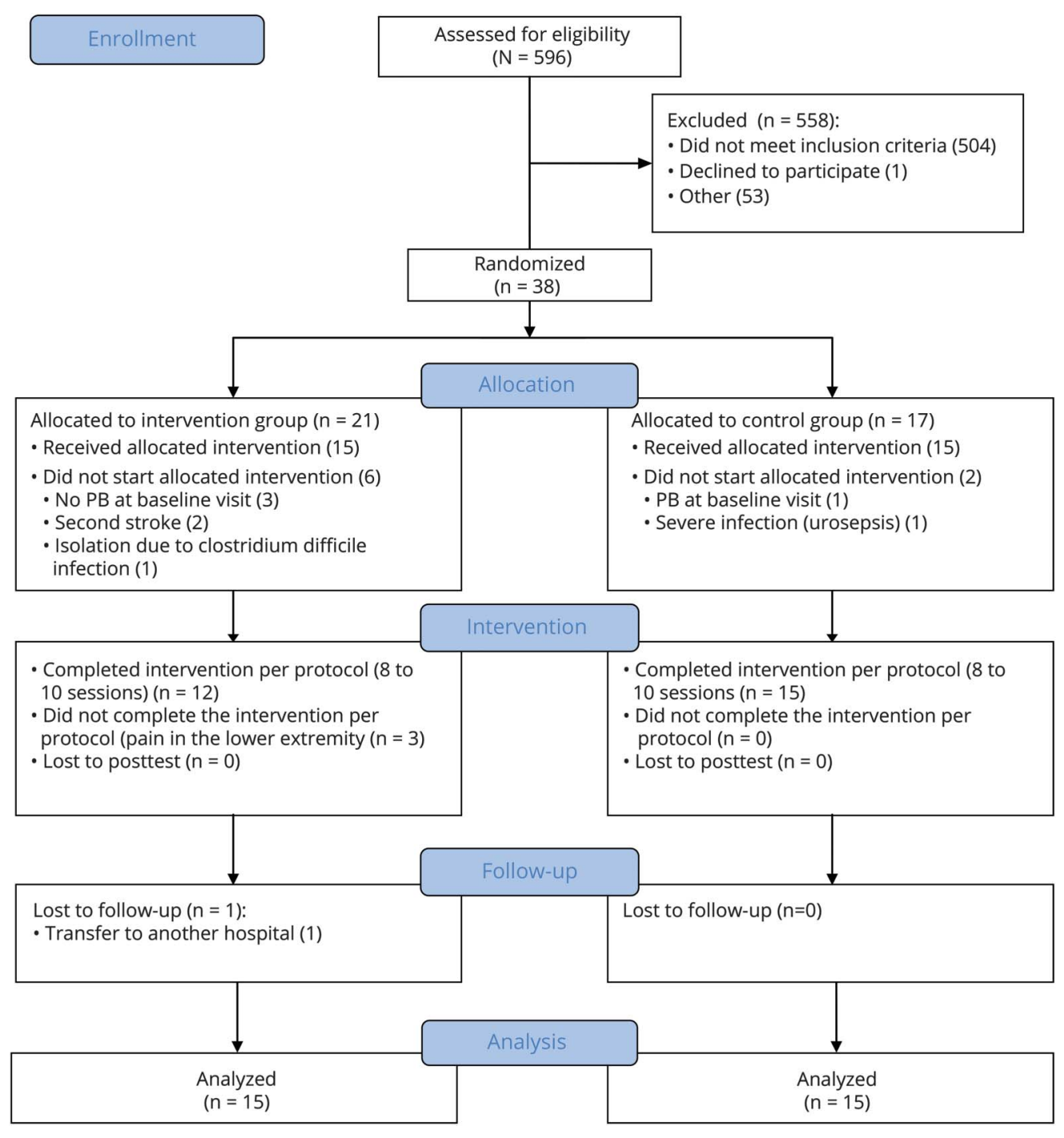

$\mathrm{PB}=$ pusher behavior. 
Table 1 Demographics and baseline clinical characteristics of the intervention group (RAGT) and the control group (nR-PT)

\begin{tabular}{llll}
\hline & RAGT $(\mathbf{n}=\mathbf{1 5})$ & $\mathbf{n R - P T}(\mathbf{n}=\mathbf{1 5})$ & Between-group comparisons \\
\hline Age, mean \pm SD, $\mathbf{y}$ & $72 \pm 9$ & $71 \pm 10$ & $t_{28}=-0.500, p=0.621$ \\
\hline Sex, F/M & $5 / 10$ & $8 / 7$ & $X^{2}{ }_{1}=1.222, p=0.269$ \\
\hline Ischemic/hemorrhagic stroke & $8 / 7$ & $9 / 6$ & $X^{2}{ }_{1}=0.136, p=0.713$ \\
\hline Side of lesion, R/L & $11 / 4$ & $12 / 3$ & $X^{2}{ }_{1}=0.186, p=0.666$ \\
\hline Time since stroke, mean \pm SD, wk & $7.5 \pm 2.6$ & $8.0 \pm 3.8$ & $t_{28}=-0.436, p=0.666$ \\
\hline ACE-R, median (Q1-Q3) & $66(51-76)(n=13)$ & $57(39-64)(n=9)$ & $U=34.000, Z=-1.639, p=0.110$ \\
\hline
\end{tabular}

Abbreviations: ACE-R = Addenbrooke's Cognitive Examination-Revised; nR-PT = nonrobotic physiotherapy; Q1 = first quartile; Q3 = third quartile; RAGT = robot-assisted gait training.

\section{Results}

Thirty-eight patients were enrolled in the study between December 2011 and July 2016. The final follow-up test was made in August 2016. A flow diagram shows how patients progressed through the study, including reasons for dropping out or data lost (figure). A total of 30 patients received the allocated intervention and were included in the analysis.

There were no statistically significant differences between the intervention and the control groups for any of the demographic or clinical characteristics at the beginning of the intervention (table 1). Two patients in the intervention group and 6 patients in the control group were not able to complete the ACE- $R$ because of severe cognitive or language deficits. There was no correlation between the ACE-R scores and the changes in pusher behavior over the study period (tau $\leq$ $-0.095 ; p \geq 0.5$ ).

Characteristics of the therapies, such as the number of performed therapies, actual walking or therapy time, etc., can be found in table 2.

\section{Primary outcome measures}

At baseline, there were no significant differences between groups for the SCP and the BLS (SCP: $U=105.00, r=-0.06$, $p=0.775$; BLS: $U=99.00, r=-0.10, p=0.595)$. Short-term (baseline - post) and long-term (baseline - follow-up) changes in the primary outcome measures are shown in table 3. The SCP revealed a significantly larger short-term effect and long-term effect in the intervention than in the control group. With the BLS, only the short-term effect significantly differed between groups $(U=47.50, r=-0.50, p=0.003)$, but the long-term effect did not reach significance $(U=76.50, r=$ $-0.28, p=0.069$ ).

Table 4 shows group values for all outcome measures including within-group comparisons. Both groups significantly improved pusher behavior during the study period. Wilcoxon tests revealed for the intervention group a significant improvement from baseline to post test measured with the BLS $(Z=-3.305$, $r=-0.85, p=0.001)$, and significant improvement from baseline to follow-up test measured with the SCP $(Z=-2.534$, $r=-0.65, p=0.012)$ and the BLS $(Z=-3.248, r=-0.84, p=$ $0.001)$. For the control group, a significant improvement was only found from baseline to follow-up test measured with the SCP $(Z=-2.524, r=-0.65, p=0.012)$ and the BLS $(Z=$ $-2.598, r=-0.67, p=0.009$ ).

Table 2 Characteristics of the therapeutic interventions in the intervention group (RAGT) and the control group (nR-PT)

Mean \pm SD

(min-max)

\section{RAGT (n = 15)}

\begin{tabular}{ll}
\hline No. of therapy sessions & $8.1 \pm 2.6(1-10)$ \\
\hline Walking time, $\mathrm{min}$ & $19.2 \pm 6.0(3-26)$ \\
\hline Walking speed, km/h & $2.1 \pm 0.4(1.1-2.7)$ \\
\hline Body weight support (\% body weight) & $\begin{array}{l}38.6 \pm 18.0 \\
(5.6-57.5)\end{array}$ \\
\hline
\end{tabular}

nR-PT $(n=15)$

\begin{tabular}{ll}
\hline No. of therapy sessions & $9.7 \pm 0.5(9-10)$ \\
\hline Active therapy time per session, min & $32 \pm 14(16-58)$ \\
\hline Sitting, min/session & $10 \pm 7(0-24)$ \\
\hline Sitting to standing, min/session & $6 \pm 4(1-16)$ \\
\hline Transferring, min/session & $6 \pm 3(2-14)$ \\
\hline $\begin{array}{l}\text { Standing, min/session } \\
\text { No. of sessions in which external references } \\
\text { were used }\end{array}$ & $7.2 \pm 1.9(4-10)$ \\
\hline $\begin{array}{l}\text { No. of sessions with cotherapy (2 therapists) } \\
\text { Ner }\end{array}$ & $3.3 \pm 1.8(1-6)$ \\
\hline
\end{tabular}

Abbreviations: RAGT = robot-assisted gait training; min-max = minimummaximum; nR-PT = nonrobotic physiotherapy.

Values were first averaged for the individual patient and then for the group. 
Table 3 Median change (Q1-Q3) in primary outcome measures from baseline

\begin{tabular}{|c|c|c|c|c|c|c|}
\hline & \multicolumn{3}{|c|}{ Difference, baseline - post } & \multicolumn{3}{|c|}{ Difference, baseline - follow-up } \\
\hline & RAGT $(n=15)$ & $n R-P T(n=15)$ & $\begin{array}{l}\text { Between-group } \\
\text { comparison }\end{array}$ & RAGT $(n=15)$ & nR-PT (n = 15) & $\begin{array}{l}\text { Between-group } \\
\text { comparison }\end{array}$ \\
\hline SCP & $\begin{array}{l}-0.5(-3.50 \text { to } \\
0.00)\end{array}$ & $\begin{array}{l}0.0(0.00 \text { to } \\
-0.25)\end{array}$ & $U=69.00, r=-0.33, p=0.037$ & $\begin{array}{l}-2.0(-3.25 \text { to } \\
-1.00)\end{array}$ & $\begin{array}{l}-0.5(-2.25 \text { to } \\
0.00)\end{array}$ & $U=54.00, r=-0.44, p=0.008$ \\
\hline BLS & $-4(-6$ to -1$)$ & $0(-2$ to -1$)$ & $U=47.50, r=-0.50, p=0.003$ & $-4(-6$ to -3$)$ & $-3(-5$ to -1$)$ & $U=76.50, r=-0.28, p=0.069$ \\
\hline
\end{tabular}

Abbreviations: BLS = Burke Lateropulsion Scale; nR-PT = nonrobotic physiotherapy; Q1 = first quartile; Q3 = third quartile; RAGT = robot-assisted gait training; $\mathrm{SCP}=$ Scale for Contraversive Pushing.

The number of patients not showing pusher behavior according to the SCP at post test significantly differed between the groups $\left(\chi_{1}^{2}=4.658, p=0.031\right)$ : The behavior ceased in 6 of 15 patients $(40.0 \%)$ in the intervention group and in 1 of 15 patients $(6.7 \%)$ of the control group during the intervention phase. At follow-up test, 9 of 15 patients (60.0\%) in the intervention group and 5 of 15 patients (33.4\%) in the control group showed no pusher behavior $\left(\chi_{1}^{2}=2.143, p=\right.$ $0.143)$.

\section{Secondary outcome measures}

Group values and within-group comparisons for the POMA, the FAC, and the SVV are shown in table 4. There were no significant differences between groups for the POMA and the FAC at baseline (POMA: $U=109.500, Z=-0.145, r=-0.03$, $p=0.902$; FAC: $U=105.00, Z=-1.000, r=-0.18, p=0.775)$. The intervention group showed a significantly larger longterm effect with the POMA than the control group ( $U=$ 72.000, $Z=-1.802, r=-0.33, p=0.049)$. The short-term effect was similar between groups $(U=99.000, Z=-0.803, r=$ $-0.15, p=0.298)$. Changes in the FAC did not differ between the groups (short-term effect: $U=112.00, Z=0.000, r=-0.00$, $p=0.500$; long-term effect: $U=102.00, Z=-0.590, r=-0.11$, $p=0.342$ ). Only 9 patients of the intervention group and 8 patients of the control group were able to perform SVV assessment. Neither the SVV at baseline $(U=25.000, Z=-1.060$, $r=-0.26, p=0.321)$ nor the SVV changes significantly differed between the groups $(U=29.000, Z=-0.682, r=-0.17, p=$ $0.541 ; U=35.000, Z=-0.096, r=-0.02, p=0.963)$.

\section{Discussion}

The present study investigated the effectiveness of repeated RAGT on pusher behavior. Our results indicate that 2 weeks of intensive RAGT leads to a statistically significant reduction of pusher behavior compared to nR-PT. The beneficial effect of RAGT persisted 2 weeks after the end of the intervention phase when using the SCP. RAGT led not only to a larger improvement of pusher behavior, but also to a larger number of patients whose pusher behavior ceased completely. Forty percent and $60 \%$ of patients of the intervention group showed no more pusher behavior after the intervention phase and at the follow-up, respectively. In contrast, $7 \%$ and $33 \%$ of patients of the control group recovered from pusher behavior at the respective time points. Recovery of pusher behavior is very relevant for the treatment and outcome of these patients. The longer pusher behavior persists, the worse is the rehabilitation outcome. ${ }^{8}$ However, a reduction of the behavior also seems important, as the severity of pusher behavior correlates with the deviation of the inner reference of verticality and with the impairment of balance performance. ${ }^{1,26}$ Consequently, improvements in pusher behavior might facilitate the mobilization and treatment of patients. Similar to this, we found a statistically significant larger improvement of balance performance in the intervention group than in the control group at follow-up testing. No differences between groups were found for the FAC and SVV. In both groups, SVV was within the ranges of normality $\left(-2.5^{\circ} \text { to } 2.5^{\circ}\right)^{3}$ at baseline testing and remained within this range over the study period. The FAC, by contrast, resulted in floor effects in our study sample, as gait is usually not possible for patients with pusher behavior, except in very mild pusher behavior. Although the intervention group practiced gait and the control group mainly postural control, walking abilities remained very limited in both groups.

RAGT is an active and task-oriented treatment approach in which the patient's body is in an earth vertical position, as suggested previously for patients with pusher behavior. ${ }^{4}$ The harness system and the firm connection to the robot might lessen the patient's fear of falling and it secures and supports the patient in an upright standing position. ${ }^{27}$ This allows training in an upright body position for a prolonged period of time. In the present study, patients spent on average 19 minutes per session walking in the gait trainer. When adding the time for pre- and postprocessing (attachment and removal of the harness and the cuffs) and for possible breaks, patients of the intervention group spent approximately 30 to 40 minutes per session in an upright standing position. According to the therapy protocols, patients of the control group trained on average only 11 minutes per session in a standing position.

We suggest that not only the upright body orientation but especially locomotion in that position are beneficial to treat pusher behavior. RAGT allows patients to practice weight loading and unloading of the lower extremities. The patient 
can learn to place weight on the paretic limb and simultaneously move the nonparetic leg in a rhythmic pattern while maintaining an upright body position. ${ }^{4,13}$ The guided walking pattern also induces a stereotyped somatosensory cueing and an increased somatosensory stimulation, which might improve the altered internal reference of verticality. ${ }^{1,28,29}$

In addition, patients must deal with conflicting sensory information during RAGT. While the proprioceptive inputs from the lower extremities mimic an appropriate stepping pattern on a moving support surface, the vestibular and visual inputs do not imply forward movement in space. Conflicting sensory information may lead to a continuous reweighting of the sensory input. This training of the central sensory information processing might help to improve the impaired body orientation in space and postural stability. ${ }^{30}$ It must still be determined which of the discussed factors are relevant, also whether alone or in combination, for the improvement of pusher behavior. It would be interesting to compare RAGT with body-weight-supported gait training on the treadmill or on the ground. So far, gait training is not a common approach in the treatment of pusher behavior, because these patients have a severely impaired postural control, and improvement of gait is rarely a therapeutic objective. However, this study showed that RAGT also affects pusher behavior and postural control.

Another advantage of RAGT might be that its effects on pusher behavior are based on a mostly implicit learning process while other therapeutic approaches, such as physiotherapy with visual feedback training, are based on an explicit learning process. Explicit motor learning involves cognitive stages within the learning process and is dependent on working memory involvement, while implicit learning requires no or only minimal conscious involvement and only minimally loads working memory. ${ }^{31}$ During visual feedback training, the patient is instructed to visually explore the surrounding and has to recognize and understand the mismatch between their perceived and their actual body orientation. After the patient realizes their tilted posture, they have to learn postural reactions to align their body with the "true vertical," i.e., the vertical visual cues. ${ }^{9}$ Finally, the patient has to automatize this strategy and transfer it into everyday situations. Because patients with pusher behavior often show severe cognitive impairments, ${ }^{12,13}$ the visual feedback therapy might be unfeasible for many of them. In addition, it is based on an unimpaired perception of the visual vertical. There is no specific bias of the SVV in patients with pusher behavior, but they show a higher threshold in visual verticality perception than patients with stroke who do not have pusher behavior. ${ }^{32}$ So far, evidence on the effectiveness of visual feedback training in patients with pusher behavior is insufficient. ${ }^{9-11}$

In the present study, we used 2 different scales to evaluate pusher behavior, namely, the SCP and the BLS. Both scales revealed a larger reduction of pusher behavior after 2 weeks of RAGT compared to nR-PT. After the intervention phase, 
pusher behavior further improved according to the SCP, but only slightly changed on the BLS. This is surprising since the BLS is considered to be more sensitive than the SCP in detecting changes in pusher behavior. ${ }^{17,33}$ Our data revealed some incongruences between the scales and the measurements mainly for the items rating resistance during sitting and the supine item of the BLS. Previously, researchers reported certain difficulties in detecting small body tilts or determining the degree of tilt using the BLS, and some differences between the rating of spontaneous body posture on the SCP and the actual body posture assessed with photography. ${ }^{26,33}$ Although the investigators of the present study were experienced and trained in the use of the scales, problems in the assessment might possibly explain the incongruences.

Our study had certain limitations regarding the generalization of the results. Most of the exclusion criteria of the present study, such as orthostatic intolerance or acute cardiovascular disease, were contraindications for the training with the Lokomat defined by the company. While training with the device is restricted to a certain patient population because of these contradictions, nR-PT can be used with almost any patient. Dependent on the severity of pusher behavior and the therapy content, cotherapy ( 2 therapists) might facilitate $n R-$ $\mathrm{PT}$. We found cotherapies most frequently in patients with moderate to severe pusher behavior (SCP scores $>3$ ). For several patients of the intervention group, it was not possible to comply with the therapy protocol (e.g., failure of therapy session or less than the targeted walking time). This implies that the intensive therapy setting used in the present study might not always be feasible. Future research should determine whether a less intensive setting has similar effects. In addition, a longer intervention phase might lead to a better outcome and possibly to improved long-term effects. The long-term effect in this study was calculated as the difference between baseline and follow-up test. Because the follow-up period lasted only 2 weeks, the so-called long-term effect has to be interpreted with caution. A longer follow-up period would result in more valid results about the long-term effects of RAGT and nR-PT.

All patients included in this study showed cognitive deficits with ACE-R scores <84 (age-dependent normal range 84-100). ${ }^{14}$ Several patients had severe cognitive deficits and 8 patients were not able to do the cognitive examination. Even though we found no correlation between the ACE-R score and the improvement of pusher behavior, these deficits might have influenced the patients' response to the interventions. This applies in particular to the control group in which the deficits were more pronounced and the intervention involved more explicit learning processes. The 3 patients of the intervention group who did not complete the study per protocol had relatively high ACE-R scores (73-79). We did not apply the cognitive deficits as an exclusion or a stratification criterion. In future studies, it is advised to control more thoroughly for cognitive function and its influence on patients' adherence and response to treatment.
Finally, the "natural" recovery has to be considered. A previous study showed that the recovery of pusher behavior varies widely among patients: approximately half of the patients identified with pusher behavior at rehabilitation admission exhibited the behavior less than 4 weeks during their rehabilitation stay, while others showed very persistent pusher behavior. ${ }^{8}$ A delayed recovery of pusher behavior seems to be associated with age and severe stroke impairments. ${ }^{12}$ In the present study, the average time between stroke onset and study inclusion was about 8 weeks in both groups (minimum 3 weeks), and demographic characteristics did not show any differences between groups. Future intervention trials should consider prognostic factors in their study design and mainly target patients with risk factors for developing persistent pusher behavior in order to ameliorate their rehabilitation outcome.

Two weeks of RAGT seems to persistently reduce pusher behavior. The forced upright body position during locomotion and the somatosensory stimulation might recalibrate the disturbed inner reference of verticality in patients with pusher behavior. Previous work showed that these patients require prolonged rehabilitation. ${ }^{5,8}$ Consequently, efficient approaches are needed to improve their rehabilitation. Our results suggest that the recovery process is more efficient when RAGT is applied.

\section{Author contributions}

J.B., C.K., F.M., and K.J. contributed to the conception of the study. J.B. and C.K. were involved in data acquisition, analysis, and interpretation. J.B., C.K., F.M., and K.J. critically revised the manuscript for important intellectual content. J.B. and C.K. are responsible for the statistical analysis. F.M. and K.J. supervised the study and obtained funding.

\section{Acknowledgment}

The authors thank Silke Heller and the team of physiotherapists for assisting with the therapies, Petra Bauer and Tim Große for helping with patient recruitment and data collection, and J. Benson for copyediting the manuscript.

\section{Study funding}

This work was supported by funds from the German Federal Ministry of Education and Research (BMBF IFB 01EO0901).

\section{Disclosure}

The authors report no disclosures relevant to the manuscript. Go to Neurology.org/N for full disclosures.

\section{Publication history}

Received by Neurology November 24, 2017. Accepted in final form July 2, 2018.

\section{References}

1. Bergmann J, Krewer C, Selge C, Müller F, Jahn K. The subjective postural vertical determined in patients with pusher behavior during standing. Top Stroke Rehabil 2016;23:184-190.

2. Karnath HO, Ferber S, Dichgans J. The origin of contraversive pushing: evidence for a second graviceptive system in humans. Neurology 2000;55:1298-1304. 
3. Pérennou DA, Mazibrada G, Chauvineau V, et al. Lateropulsion, pushing and verticality perception in hemisphere stroke: a causal relationship? Brain 2008;131: 2401-2413.

4. Davies PM. Steps to Follow: A Guide to the Treatment of Adult Hemiplegia. New York: Springer; 1985.

5. Pedersen PM, Wandel A, Jorgensen HS, Nakayama H, Raaschou HO, Olsen TS. Ipsilateral pushing in stroke: incidence, relation to neuropsychological symptoms, and impact on rehabilitation. The Copenhagen Stroke Study. Arch Phys Med Rehabil 1996;77:25-28.

6. Abe H, Kondo T, Oouchida Y, Suzukamo Y, Fujiwara S, Izumi S. Prevalence and length of recovery of pusher syndrome based on cerebral hemispheric lesion side in patients with acute stroke. Stroke 2012;43:1654-1656.

7. Baccini M, Paci M, Nannetti L, Biricolti C, Rinaldi LA. Scale for Contraversive Pushing: cutoff scores for diagnosing "pusher behavior" and construct validity. Phys Ther 2008;88:947-955.

8. Krewer C, Luther M, Müller F, Koenig E. Time course and influence of pusher behavior on outcome in a rehabilitation setting: a prospective cohort study. Top Stroke Rehabil 2013;20:331-339.

9. Broetz D, Johannsen L, Karnath HO. Time course of "pusher syndrome" under visual feedback treatment. Physiother Res Int 2004;9:138-143.

10. Paci M, Nannetti L. Physiotherapy for pusher behaviour in a patient with post-stroke hemiplegia. J Rehabil Med 2004;36:183-185.

11. Krewer C, Riess K, Bergmann J, Müller F, Jahn K, Koenig E. Immediate effectiveness of single-session therapeutic interventions in pusher behaviour. Gait Posture 2013;37: 246-250.

12. Babyar SR, Peterson MG, Reding M. Case-control study of impairments associated with recovery from "pusher syndrome" after stroke: logistic regression analyses. J Stroke Cerebrovasc Dis 2017;26:25-33.

13. Premoselli S, Cesana L, Cerri C. Pusher syndrome in stroke: clinical, neuropsychological and neurophysiological investigation. Eur Med Phys 2001;37: $143-151$.

14. Mioshi E, Dawson K, Mitchell J, Arnold R, Hodges JR. The Addenbrooke's Cognitive Examination Revised (ACE-R): a brief cognitive test battery for dementia screening. Int J Geriatr Psychiatry 2006;21:1078-1085.

15. Baccini M, Paci M, Rinaldi LA. The Scale for Contraversive Pushing: a reliability and validity study. Neurorehabil Neural Repair 2006;20:468-472.

16. D'Aquila MA, Smith T, Organ D, Lichtman S, Reding M. Validation of a lateropulsion scale for patients recovering from stroke. Clin Rehabil 2004;18:102-109.

17. Babyar SR, Peterson MG, Bohannon R, Pérennou D, Reding M. Clinical examination tools for lateropulsion or pusher syndrome following stroke: a systematic review of the literature. Clin Rehabil 2009;23:639-650.
18. Tinetti ME. Performance-oriented assessment of mobility problems in elderly patients. J Am Geriatr Soc 1986;34:119-126.

19. Holden MK, Gill KM, Magliozzi MR, Nathan J, Piehl-Baker L. Clinical gait assessment in the neurologically impaired: reliability and meaningfulness. Phys Ther 1984;64:35-40.

20. Mehrholz J, Wagner K, Rutte K, Meissner D, Pohl M. Predictive validity and responsiveness of the Functional Ambulation Category in hemiparetic patients after stroke. Arch Phys Med Rehabil 2007;88:1314-1319.

21. Zwergal A, Rettinger N, Frenzel C, Dieterich M, Brandt T, Strupp M. A bucket of static vestibular function. Neurology 2009;72:1689-1692.

22. Noether GE. Sample size determination for some common nonparametric tests. J Am Stat Assoc 1987;82:645-647.

23. Walters SJ. Sample size and power estimation for studies with health related quality of life outcomes: a comparison of four methods using the SF-36. Health Qual Life Outcomes 2004;2:26.

24. Rosenthal R, Rosnow RL, Rubin DB. Contrasts and Effect Sizes in Behavioral Research: A Correlational Approach. Cambridge: Cambridge University Press; 2000.

25. Cohen J. A power primer. Psychol Bull 1992;112:155-159.

26. Clark E, Hill KD, Punt TD. Responsiveness of 2 scales to evaluate lateropulsion or pusher syndrome recovery after stroke. Arch Phys Med Rehabil 2012;93:149-155.

27. Shepherd RB, Carr JA. New aspects for the physiotherapy of pushing behaviour, D. Broetz and H.-O. Karnath,Neurorehabilitation 20 (2005), 133-138. Neurorehabilitation 2005;20:343-345.

28. Barra J, Marquer A, Joassin R, et al. Humans use internal models to construct and update a sense of verticality. Brain 2010;133:3552-3563.

29. Pérennou D, Piscicelli C, Barbieri G, Jaeger M, Marquer A, Barra J. Measuring verticality perception after stroke: why and how? Neurophysiol Clin 2014;44:25-32.

30. Krewer C. Are therapeutic effects on pusher behaviour based on conflicting sensory information? Presented at the international conference Multisensory Motor Behavior: Impact of Sound; September 30, 2013, and October 1, 2013; Hannover.

31. Kleynen M, Braun SM, Bleijlevens MH, et al. Using a Delphi technique to seek consensus regarding definitions, descriptions and classification of terms related to implicit and explicit forms of motor learning. PLoS One 2014;9:e100227.

32. Paci M, Matulli G, Megna N, Baccini M, Baldassi S. The Subjective Visual Vertical in patients with pusher behaviour: a pilot study with a psychophysical approach. Neuropsychol Rehabil 2011;21:539-551.

33. Bergmann J, Krewer C, Riess K, Müller F, Koenig E, Jahn K. Inconsistent classification of pusher behaviour in stroke patients: a direct comparison of the Scale for Contraversive Pushing and the Burke Lateropulsion Scale. Clin Rehabil 2014;28: 696-703. 